\title{
Omega-3 polyunsaturated fatty acids as an angelus custos to rescue patients from NSAID-induced gastroduodenal damage
}

\author{
Jong Min Park • Young Min Han • Migyeong Jeong • \\ Eun Hee Kim • Weon Jin Ko $\cdot$ Joo Young Cho • \\ Ki Baik Hahm
}

Received: 24 November 2014/ Accepted: 17 December 2014/Published online: 13 January 2015

(C) Springer Japan 2015

\begin{abstract}
Nonsteroidal anti-inflammatory drugs (NSAIDs) are one of the drug types frequently prescribed for their analgesic, anti-inflammatory, and antithrombotic actions, but carry a risk of major gastroduodenal damage from mild erosive changes to serious ulceration leading to fatal outcomes. From the long history of willow tree bark and its extracts being applied for the relief of pain and fever, the synthesis of acetylsalicylic acid, the development of selective cyclooxygenase 2 inhibitors (coxibs), and the identification of a G-protein-coupled receptor for prostaglandin, the popular combination regimen of an NSAID and a proton pump inhibitor was invented, but development was continued for further improvement. With regard to major NSAID adverse effects, gastrointestinal (GI) and cardiovascular $(\mathrm{CV})$ risks still remained as problems to be solved. In this review, it is shown that $n-3$ polyunsaturated fatty acid (PUFA) based NSAIDs can be an angelus custos, supported with facts that an intake of essential $n$-3 PUFAs
\end{abstract}

Part of this review was presented at The 4th International Forum of the 100th General Meeting of the Japanese Society of Gastroenterology.

J. M. Park · Y. M. Han · M. Jeong · E. H. Kim ·

K. B. Hahm (ه)

CHA University Cancer Prevention Research Center,

CHA Bio Complex, 335 Pangyo-ro, Gundang-gu,

Seongnam 463-400, Korea

e-mail: hahmkb@cha.ac.kr

J. M. Park

e-mail: jmpark@cha.ac.kr

W. J. Ko · J. Y. Cho · K. B. Hahm

Department of Gastroenterology, CHA University

Bundang Medical Center, 59 Yatap-ro, Bundang-gu,

Seongnam 463-712, Korea orchestrates concerted protective actions against two notorious side effects of NSAIDs, the aforementioned GI risk and CV risk of NSAIDs. Since pills containing $n-3$ PUFAs, omega-3-acid ethyl ester capsules (Lovaza, Omarcor), have already been safely prescribed to prevent atherosclerosis through lessening lipid burdening, the introduction of a drug delivery system such as a gastroretentive form of $n$-3 PUFA based NSAIDs will highlight newer hope for GI safety under the guarantee of reduced CV risk. Because $n-3$ PUFAs have been proven to attenuate cytotoxicity, inhibit lipid-raft-associated harmful signaling, and relieve oxidative stress relevant to NSAIDs, $n$-3 PUFA based NSAIDs will be next-generation GI-safe NSAIDs.

Keywords Nonsteroidal anti-inflammatory drug · Gastrointestinal risk $\cdot n-3$ polyunsaturated fatty acid $\cdot$ Lipid raft $\cdot n-3$ polyunsaturated fatty acid based nonsteroidal anti-inflammatory drug

$\begin{array}{ll}\text { Abbreviations } \\ \text { COX } & \text { Cyclooxygenase } \\ \text { Coxib } & \text { Cyclooxygenase } 2 \text { inhibitor } \\ \text { CV } & \text { Cardiovascular } \\ \text { DHA } & \text { Docosahexaenoic acid } \\ \text { EPA } & \text { Eicosapentaenoic acid } \\ \text { ER } & \text { Endoplasmic reticulum } \\ \text { GI } & \text { Gastrointestinal } \\ \text { LTB }_{4} & \text { Leukotriene } \text { B }_{4} \\ \text { NSAID } & \text { Nonsteroidal anti-inflammatory drug } \\ \text { PGE }_{2} & \text { Prostaglandin } E_{2} \\ \text { PGI }_{2} & \text { Prostacyclin } \\ \text { PPI } & \text { Proton pump inhibitor } \\ \text { PUFA } & \text { Polyunsaturated fatty acid } \\ \text { ROS } & \text { Reactive oxygen species } \\ \text { RvD1 } & \text { Resolvin D1 }\end{array}$


Unmet medical need regarding adverse effects associated with nonsteroidal anti-inflammatory drugs

Although recent studies have shown a profound decrease in the number of hospitalizations due to upper gastrointestinal (GI) tract complications owing to widespread use of proton pump inhibitors (PPIs), safer nonsteroidal anti-inflammatory drug (NSAID) prescription including cyclooxygenase (COX)-2 inhibitors (coxibs), combination with some gastroprotective medications, and decreased prevalence of Helicobacter pylori infection, improvement is still mandatory to achieve higher GI safety, lower cardiovascular (CV) risk, and rescue of patients from renal complications [1]. With inhibition of COX-1 with aspirin or ibuprofen, NSAIDs result in degradation of the protective mucous layer of the stomach and induce various degrees of damage ranging from relatively mild nausea and epigastric pain to severe perforation and bleeding ulcer [2], as well as various degrees of $\mathrm{CV}$ and renal toxicity. Naproxen is the best option in patients with high CV risk, but imparts moderate GI risk. On the other hand, patients taking aspirin are a great challenge for treatment, since interaction with frequently prescribed NSAIDs such as ibuprofen and naproxen may alter the antiplatelet effect of aspirin, representing a potential clinical problem of increased risk of GI bleeding. Changing NSAID treatment to diclofenac or celecoxib may also not be an option in patients with a history CV events [3]. Coxibs reduce endothelial production of prostacyclin $\left(\mathrm{PGI}_{2}\right)$, while leaving the platelet production of thromboxane $\mathrm{A} 2$ intact, by which increasing degrees of selectivity for COX-2 are associated with augmented $\mathrm{CV}$ risk and inversely decreasing degrees of selectivity for COX-2 (COX-1 inhibitor) are associated with augmented GI risk. Although risk evaluation should be considered before the use of safer and efficacious NSAIDs in the clinic at the individual patient level, in practice, since different clinical scenarios involving these three factors associated with the presence of different GI and $\mathrm{CV}$ risk factors exist, the best solution is tailoring each individual GI and CV risk [4]. In the following sections, we give a brief description of adverse effects of nonselective NSAIDs.

GI risk

Although whether NSAID intake in the presence of H. pylori infection may further increase the risk of peptic ulcer caused by the presence of only one risk factor is still a matter of debate, $H$. pylori infection, NSAID use, or lowdose aspirin use increased the risk of development of peptic ulcer as well as GI complications significantly and independently [5]. With regard to gastric damage, NSAIDinduced enteropathy has gained much attention in the last few years with the introduction of capsule endoscopy and enteroscopy. NSAIDs increase the risk of lower GI tract bleeding and perforation to a similar extent as that seen in the upper GI tract, and coxibs have the same beneficial effects as nonselective NSAIDs but with less GI toxicity in the upper GI tract and probably in the lower GI tract, whereas PPI combination aggravated intestinal damage due to changes in gut microbiota [6, 7]. In spite of these limitations, the strategy of choice for GI risk reduction with NSAIDs is the combination of conventional NSAIDs with PPIs or coxibs because they were reported to reduce the risk [8]. In the pursuit of greater safety under guaranteed benefits of NSAIDs, the development of NO-releasing, $\mathrm{H}_{2} \mathrm{~S}$-releasing, and phosphatidylcholine-associated NSAIDs is continuing, but is still in the preclinical stage.

\section{CV risk}

The discovery of two COX isoforms-COX-1 constitutively expressed in normal tissues, and COX-2 induced at sites of inflammation-led to the development of coxibs with the hope of significantly reducing the GI toxicity mentioned before. However, increased knowledge of the physiological roles of COX-2 in a variety of tissues, especially after COX-2 knockout mice showed renal and $\mathrm{CV}$ abnormalities, and the withdrawal of rofecoxib and valdecoxib from the market because of reported CV toxicity have challenged the benefits of coxibs [9]. NSAID usage generally imposed the risk of $\mathrm{CV}$ events including myocardial infarction or cerebrovascular accident whereas low-dose aspirin is usually used to decrease the risk of CV disease for high-risk patients [10], whereas NSAID use is associated with excess risk of death and myocardial infarction by use of coxibs. The vessel-constricting compound thromboxane A2 stimulates activation of platelets and increases platelet aggregation, and blood vessels produce the anticlotting compound $\mathrm{PGI}_{2}$. To balance the opposing actions of clotting and blood flow, COXs regulate thromboxane $\mathrm{A} 2$ and $\mathrm{PGl}_{2}$ levels during blood vessel injury. Since coxibs reduce the amounts of $\mathrm{PGI}_{2}$, they increase the risk of myocardial infarction. In addition, increases in vasoconstriction caused by coxibs can also lead to high blood pressure and renal insufficiency [11].

Renal risk and other risks

Long-term use of NSAIDs can lead to impaired renal blood flow and chronic renal damage by continuous inhibition of prostaglandins [12]. Since prostaglandins are important for proper blood vessel function within the kidneys [13], highdose NSAID therapy is associated with chronic kidney disease. NSAID-associated chronic kidney disease leads to high blood pressure, salt and water retention, and 
electrolyte imbalances [13]. The major cause of NSAIDassociated renal damage is the contribution of NSAIDs to mitochondrial dysfunction, resulting in excessive production of highly reactive free radicals and subsequent tissue damage [14]. Also, NSAIDs caused liver toxicity relevant to oxidative stress in vascular tissue [15]. Hematological complications of traditional NSAIDs are relatively rare, but NSAIDs increase the bleeding time of blood by inhibition of thromboxane A2. Consequently, the decreased platelet aggregation and the prolonged bleeding time further aggravate GI complications. Phenylbutazone use and indomethacin use are sometimes associated with agranulocytosis [16].

Safer NSAIDs to cover the aforementioned adverse effects

In addition to synthetic prostaglandins, two gaseous mediators-nitric oxide (NO) and hydrogen sulfide $\left(\mathrm{H}_{2} \mathrm{~S}\right)$ - exert protective effects in gastric mucosa. The development of NO- or $\mathrm{H}_{2} \mathrm{~S}$-releasing NSAIDs is continuing [17, 18]. NO-releasing (or NO-donating) NSAIDs have been used to inhibit NSAID-induced leukocyte adherence by release of $\mathrm{NO}$, and $\mathrm{H}_{2} \mathrm{~S}$-releasing NSAIDs have been used to inhibit NSAID-induced vasoconstriction and ischemia by release of $\mathrm{H}_{2} \mathrm{~S}$. All of these NSAIDs rescue patients from GI and $\mathrm{CV}$ risk as well as renal complications. In addition, efforts to obtain drugs able to inhibit both 5-lipoxygenase and COX, the so-called dualacting anti-inflammatory drugs [19], have continued because dual-acting anti-inflammatory drugs retain the activity of classic NSAIDs, while avoiding their main drawbacks [20]. Currently, various structural families of dual-acting anti-inflammatory drugs have been designed, and several compounds are under clinical investigation [21], although humans have taken almost 2,000 years to develop coxibs, starting from willow tree bark to the development of aspirin [22] (Figs. 1, 3).

\section{fat-1 transgenic mice producing $n-3$ polyunsaturated fatty acids exhibited protection from NSAID damage}

In 2004, Kang et al. [23] first generated fat-1 transgenic mice. These mice engineered to carry the fat- 1 gene, which encodes an $n$-3 desaturase, from the worm Caenorhabditis elegans can add a double bond into an unsaturated fatty acid hydrocarbon chain and can convert $n-6$ polyunsaturated fatty acids (PUFAs) to $n$-3 PUFAs (Fig. 2). Mammals cannot naturally produce $n$ - 3 PUFAs, and so must rely on a dietary supply_from fish, walnuts, olive oil, etc. Since fat1 transgenic mice carry an abundance of $n$-3 PUFAs but low levels of $n$-6 PUFAs in their organs and tissues in the absence of dietary $n$-3 PUFAs, this resulted in significant benefits with regard to clinical diseases and these mice emerged as a new model for omega-3 research [24], as exemplified as follows: reduced carcinogenesis and cancer prevention [25-27] and protection from metabolic diseases [28, 29], osteoarthritis [30], several ischemic injuries [31, 32], nonalcoholic steatohepatitis [33, 34], and oxidative injury [34]. Since Lim et al. [35] showed inhibition of hepatocellular carcinoma growth through blocking of $\beta$ catenin and COX-2 and these anticancer effects shared the molecular mechanisms of NSAIDs, our group hypothesized that $n$-3 PUFAs might impose NSAID-sparing effects. fat- 1 mice (Fig. 2) showed significant rescuing outcomes such that indomethacin-induced gastric ulcers as well as intestinal ulcers were significantly ameliorated. These rescuing actions of $n$-3 PUFAs were proven to be mediated through glucose-regulated protein 120 , after which blocking significantly apoptotic signals of indomethacin, scavenging oxidative stress of indomethacin, and attenuating inflammatory mediators as well as relieving ischemic conditions. As for NSAID-induced gastric ulcerations, recently much attention has been paid to intestinal injuries. Although a PPI can prevent NSAID-induced gastric ulcerations, it rather aggravated intestinal ulcerations through dysbiosis. Therefore, the emergence of $n$-3 PUFA based NSAIDs can be an escape from NSAID-induced GI damage (Fig. 3).

\section{Molecular mechanisms for how $n$-3 PUFAs rescue patients from NSAID-induced GI damage}

\section{Attenuating NSAID-associated apoptosis}

The main pharmacological action of NSAIDs is through the inhibition of prostaglandin synthesis via the suppression of COX activity, but indiscernible decreases of the levels of gastroprotective prostaglandin $\mathrm{E}_{2}\left(\mathrm{PGE}_{2}\right)$ have been regarded as a factor contributing to GI toxicity. There have been many publications explaining COX-independent mechanisms relating to GI toxicity, one of which is that NSAIDs themselves can trigger apoptosis mediated by endoplasmic reticulum (ER) stress [36]. Since ER stress prevails after administration of NSAIDs and autophagy is known as a phenomenon following ER stress, autophagic cell death can be a factor responsible for NSAID-induced GI toxicities. Autophagic cell death is inducible in response to cellular stresses, including nutrient deprivation and oxidative stress [37]. Tsutsumi et al. [36] first examined the association between ER stress response and NSAIDinduced apoptosis. Exposure of gastric mucosal cells to indomethacin induced glucose-regulated protein 78 and C/EBP homologous transcription factor as well as activating transcription factor 6 , activating transcription 
Fig. 1 Development of gastrointestinal $(G I)$-safe nonsteroidal anti-inflammatory drugs (NSAIDs): progression from willow tree bark to modern medicine. a History of development of cyclooxygenase $2(C O X-2)$ inhibitors (COXIBs) starting from ancient trepanation to willow tree bark extracts and synthesis of acetylated salicylic acids. b Double-edged sword of NSAIDs, i.e., higher GI risk, but lower cardiovascular $(\mathrm{CV})$ risk with administration of conventional nonselective NSAIDs, whereas COXIBs lower GI risk, but increase CV risk. Celecoxib, showing 4-5 of COX-2 selectivity, exhibits greater protection from either cerebral infarction risk or $\mathrm{CV}$ risk. cNSAID, COX cyclooxygenase, $C O X-1$ cyclooxygenase $1, P G$ prostaglandin

\section{A}

\section{From bark of willow tree to modern medicine} used for pain relief and antipyretics
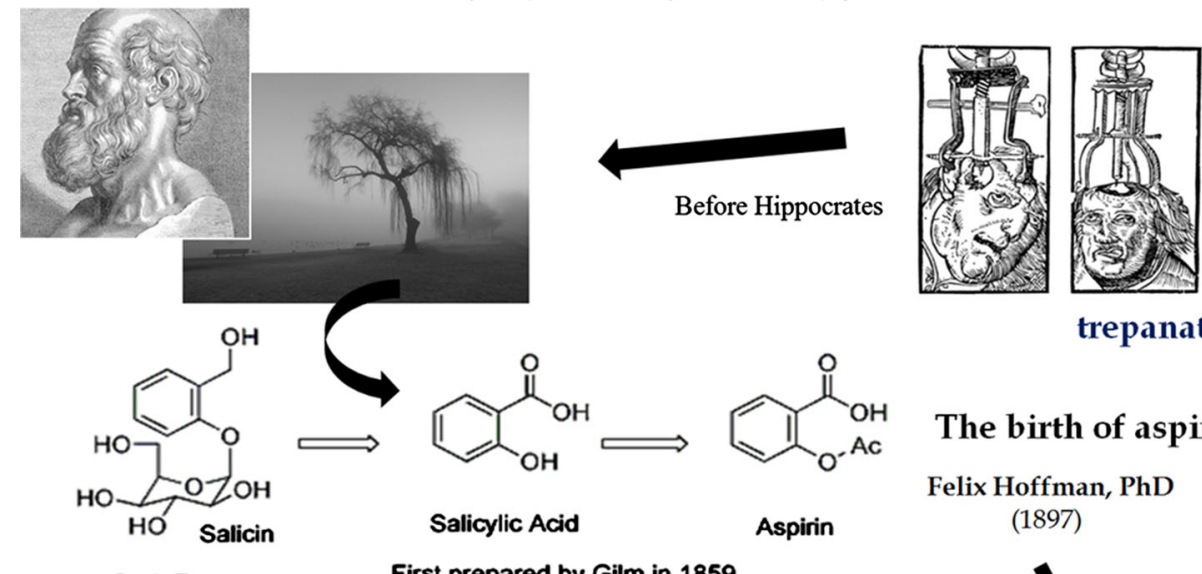

trepanation

\section{The birth of aspirin}

Felix Hoffman, PhD (1897)

Bark Extracts

First prepared by Gilm in 1859 introduced by Dreser into medicine in 1899.
cNSAID

(COX-1, COX-2 inhibitors)

\section{Discovery of PGs and COX}

John Vane, PhD (Nobel prize at 1982)
$\mathbf{B}$ GI risk (ulcer, bleeding, perforation, apoptosis in GI tract)
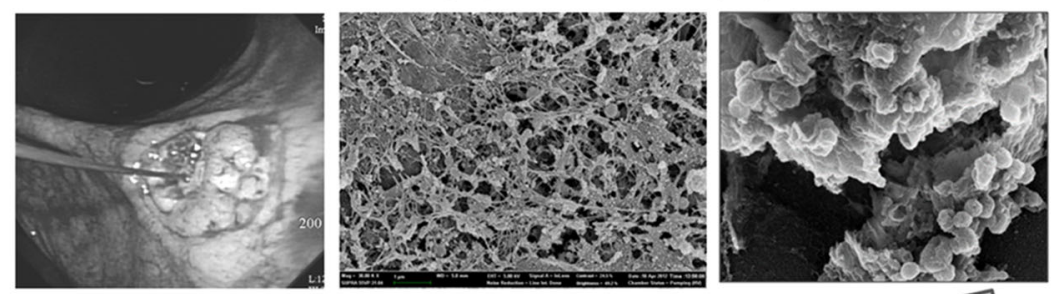

coX-1 inhibition Chronic NSAIDS

User, especially age ged person,
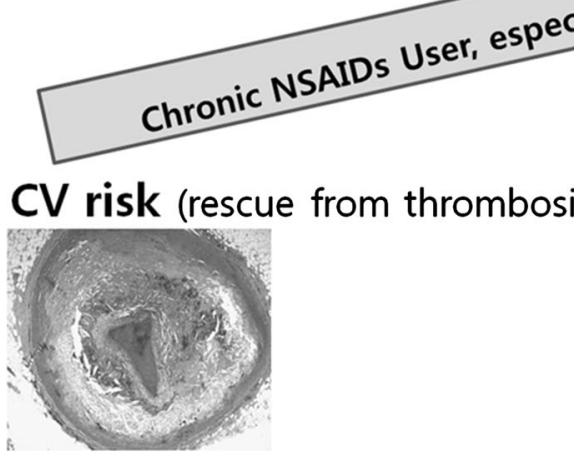

factor 4, and $\mathrm{X}$ box binding protein 1, suggesting that ER stress response seems to be generally involved in NSAID cytotoxicity irrespective of the kind of NSAID. Chen et al. [38] additionally found that the cytotoxic outcome after use of different pharmacological agents of various COX-2 inhibitory potencies was closely aligned with these agents' ability to trigger ER stress. Therefore, ER stress seems to be one of the fundamental COX-independent mechanisms of NSAID-induced gastric mucosal cytotoxicity. Another mechanism by which NSAIDs induce apoptosis is by reducing the levels of survivin, a known apoptosis inhibitor [39]. Overexpression of survivin effectively inhibited 


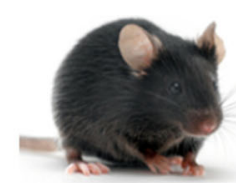

n-6 FA:

$\mathrm{CH}_{3}-\mathrm{CH}_{2}-\mathrm{CH}_{2}-\mathrm{CH}_{2}-\mathrm{CH}_{2}-\mathrm{CH}=\mathrm{CH}-\ldots-\mathrm{COOH}$

n-3 FA:

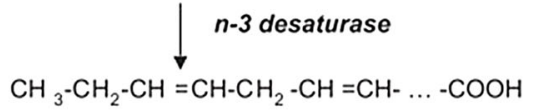

\section{Fat-1 transgenic mice}

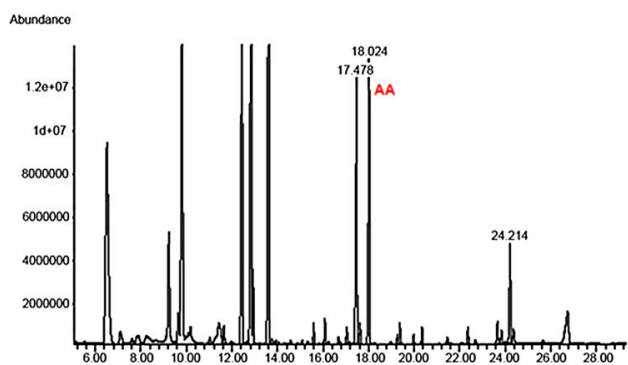

Gas Chromatography of stomach from wild type mice

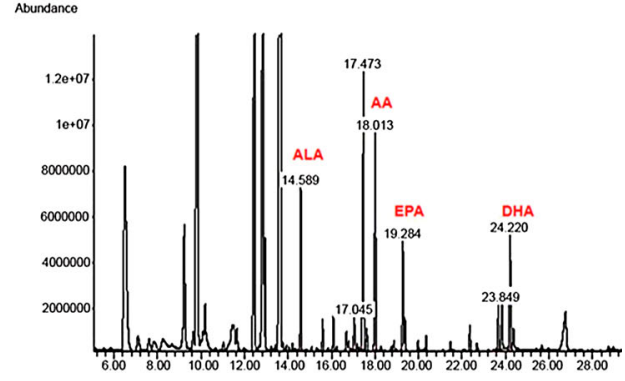

Gas Chromatography of stomach from Fat-1 TG mice
Fig. $2 n$-3 polyunsaturated fatty acids (PUFAs); essential fatty acids $(F A)$ exerting various beneficiary actions for unmet medical needs, by which fat-1 transgenic (TG) mice produced $n-3$ PUFAs through overexpressed $n-3$ desaturase. fat- 1 transgenic mice showed significant protection from indomethacin-induced gastroduodenal damage, protection from which was significantly correlated with gastric contents of $n$-3 PUFAs [ $\alpha$-linolenic acid (ALA), docosahexaenoic acid $(D H A)$, and eicosapentaenoic acid (EPA)]. On gas chromatography of stomach homogenates, $n-3$ PUFAs were generated owing to the presence of the $n-3$ desaturase transgene in fat- 1 transgenic mice, whereas they were not detected in wild-type littermates (our unpublished data). $A A$ arachidonic acid
Fig. $3 n$-3 polyunsaturated fatty acid (PUFA) based nonsteroidal anti-inflammatory drugs (NSAIDs) as nextgeneration gastrointestinal $(G I)$ safe NSAIDs.

Phosphatidylcholine $(P C)$ associated NSAIDs, NOreleasing NSAIDs, and $\mathrm{H}_{2} \mathrm{~S}$ releasing NSAIDs are all in clinical trials, and we strongly believe that $n$-3 PUFA based NSAIDs used with a gastroretentive drug delivery system $(G R D D S)$ might soon be next-generation GI-safe NSAIDs

\section{The next generation GI-safe NSAID}<smiles>[R]C(=O)OCC(COP(=O)([O-])OC[NH3+])OC([R])=O</smiles>

1) PC-NSAID
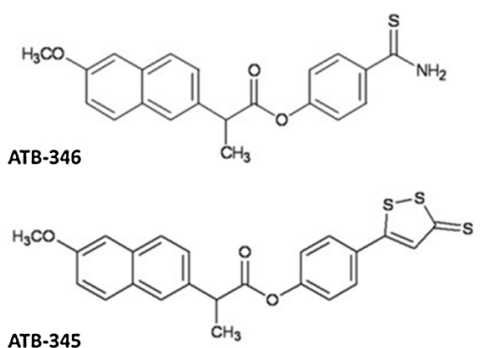

3) $\mathrm{H}_{2} \mathrm{~S}$-releasing NSAID<smiles>COc1ccc2cc(C(C)C(=O)OCCCCO[N+](=O)[O-])ccc2c1</smiles>

2) NO releasing -NSAID

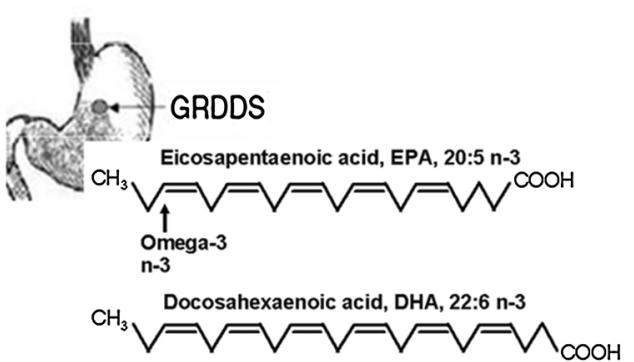

4) Gastro Retentive DDS (GRDDS) technique applied "n-3 PUFAs based NSAIDs" 
NSAID-induced apoptosis in cultured RGM-1 cells [40]. However, small interfering RNA depletion of LC3 blocked downregulation of survivin by NSIADs, indicating that survivin functions downstream of vacuole formation to regulate the sulindac sulfide induced autophagic cell death pathway [40]. NSAIDs can also mediate apoptosis by inducing the activation of caspases, a family of proapoptotic cysteine proteinases which typically exist as latent zymogens in cells. Activation of these proteins by proapoptotic signals initiates a caspase cascade whereby initiator caspases specifically activate other executioner-type caspases [41].

\section{Enhancing mucosal resolution}

It is well known that $n$-3 PUFAs have anti-inflammatory and antiapoptotic affects. Resolvins are a novel family of lipid mediators derived from eicosapentaenoic acid (EPA) and docosahexaenoic acid (DHA), both of which are $n$-3 PUFAs with anti-inflammatory and antiapoptotic effects and are classified with two distinct structural forms, the E series and the D series, respectively. In the presence of aspirin, EPA is converted by acetylated COX-2 in vascular endothelial cells to $(18 R)$-hydroperoxyeicosapentaenoic acid, which is the precursor of resolvin E1, catalyzed by 5-lipoxygenase [42] or cytochrome P450 monooxygenase [43]. DHA is converted by 15 -lipoxygenase type I to 17 -hydroxydocosahexaenoic acid, which is the precursor of the D series resolvins such as resolvin D1 (RvD1) and resolvin D2 during acute inflammation. Aspirin-acetylated COX-2 is the precursor of $(17 R)$-hydroxydocosahexaenoic acid, which is oxygenated by 5-lipoxygenase, which results in the generation of (17R)-RvD1 [44-46]. The protective effect of $n-3$ PUFAs against NSAID-induced ER stress and autophagy has not been elucidated yet. However, a recent study reported that RvD1 prevented ER-stress-induced hepatic apoptosis and lipid accumulation through the JNK-dependent pathway without inducing significant changes in the level of ER stress [47]. In addition, EPA attenuated statininduced ER stress, JNK activation, and toxicity in cultured myoblast cells, and peroxisome-proliferator-activated receptor $\delta$ may be mechanically involved in the effects of EPA [48]. Also, the administration of DHA has therapeutic potential in reducing ER stress, abnormal protein accumulation, and neurological deficits in chronic neuronal damage [49]. These results provide reliable evidence that $n$ - 3 PUFAs have cytoprotective effects through attenuation of NSAIDinduced ER stress and autophagic cell death.

Attenuating NSAID-induced inflammatory actions

Traditional NSAIDs such as aspirin and indomethacin are widely used for the relief of pain and inflammation.
However, their use is limited by their gastric toxicity. NSAID-induced gastric damage is due in large part to COX inhibition [50]. Whereas COX-1 is involved in the biosynthesis of prostaglandins that regulate mucosal blood flow and epithelial mucus and bicarbonate secretion, prostaglandins derived from COX-2 are involved in the reduction of leukocyte adherence and re-epithelialization of gastric cells [51]. Even with the introduction of coxibs, gastric injury has not been abolished [52]. In light of these findings, there is a high clinical need for therapies that prevent the gastric toxicity of NSAIDs. Gastroprotective effects of fish oil have been reported in gastric ulcers induced by ethanol [53, 54], aspirin [55, 56], indomethacin [57], dexamethasone [58], cold-restraint stress [59], and pyloric ligation [56]. Several mechanisms have been suggested to be involved in the gastroprotective effects of fish oil, including a decrease in gastric acid secretion and lipid peroxidation as well as an increase in the levels of antioxidant enzymes during pyloric ligation and cold-restraint stress in rats [56]. Although several studies have shown the gastroprotective effect of $n-3$ PUFAs and have reported some potential mechanisms behind this effect, it is less understood which pure compounds of fish oil are responsible for this effect. DHA is an $n-3$ PUFAs present in fish oil. DHA exhibits several actions, such as anti-inflammatory, neuroprotectitve, and cardioprotective effects [60, 61]. DHA also inhibits dextran sulfate sodium induced colitis in mice. Furthermore, DHA downregulates expression of tumor necrosis factor $\alpha$ and interleukin-1 $\beta$ increased by colitis [62]. Together, these findings suggest that DHA may be a pure compound of fish oil responsible for the gastroprotective actions. NSAID-induced gastric damage is due in large part to COX inhibition, which results in inhibition of synthesis of prostaglandins. Prostaglandins participate in the signal of gastric protective factors; $\mathrm{PGE}_{2}$ is involved in the increase of mucus secretion and gastric blood flow. NSAID-induced gastric mucosal injury includes inhibition of mucosal prostaglandin synthesis. It has been demonstrated that $\mathrm{PGE}_{2}$ increases mucus and bicarbonate secretion and gastric blood flow and decreases acid secretion. Thus, an inhibition of the synthesis of prostaglandins, including $\mathrm{PGE}_{2}$, by NSAIDs would block the gastroprotective actions of $\mathrm{PGE}_{2}$. Furthermore, leukotriene $\mathrm{B}_{4}\left(\mathrm{LTB}_{4}\right)$ has been implicated in NSAID-induced leukocyte adhesion [63], and inhibition of synthesis of $\mathrm{LTB}_{4}$ resulted in a significant reduction of NSAID-induced gastric injury [64, 65]. Acute DHA administration blocks the indomethacin-induced increase of $\mathrm{LTB}_{4}$ levels [65]. $n$-3 PUFAs such as DHA may exert beneficial effects by competing with $n-6$ PUFAs such as arachidonic acid for the production of lipid inflammatory mediators such as leukotriene [66]. Furthermore, it has been shown that human ingestion of fish oil leads to a 
decrease in $\mathrm{LTB}_{4}$ levels and an increase in the levels of leukotriene $\mathrm{B}_{5}$, a weak inducer of inflammation and a weak chemotactic agent [67].

\section{Enhancing host defense phase 2 enzyme response}

NSAIDs can induce damage or apoptosis by upregulating the generation of reactive oxygen species (ROS) and by inducing oxidative stress coupled with many proapoptotic signals such as nuclear factor $\mathrm{\kappa B}$ inhibition and mitogenactivated protein kinase activation [41]. However, the cellular prooxidant behavior of NSAIDs has often been the subject of controversy owing to conflicting reports that NSAIDs such as indomethacin and sulindac scavenge ROS and exert a cytoprotective and antioxidant effect in cells $[68,69]$. It has also been shown that low doses of aspirin can confer long-term cytoprotective resistance against $\mathrm{H}_{2} \mathrm{O}_{2}$-induced oxidative stress in Saccharomyces cerevisiae cells [70]. Nevertheless, it has been accepted that NSAIDs can induce the proapoptotic accumulation of ROS in mammalian cells. The molecular mechanisms underlying NSAID-induced proapoptotic generation of ROS have not yet been fully elucidated. However, it is well known that mitochondria, a major source of cellular ROS [71] and a central component of the apoptotic machinery, are profoundly affected by NSAIDs [72]. For example, the aspirin metabolite salicylate has been shown to inhibit the mitochondrial electron transport chain in mammalian cells by interacting with an $\mathrm{Fe}-\mathrm{S}$ cluster of complex I through its $o$ hydroxyl group, inducing ROS accumulation and oxidative stress, and finally causing proapoptotic events such as mitochondrial permeability transition and cytochrome $c$ release [73]. Likewise, aspirin-induced cell cycle arrest and apoptosis of HepG2 hepatoma cells were shown to be induced by ROS accumulation and increased oxidative stress, and were accompanied by severe mitochondrial dysfunction such as the inactivation of electron transport chain proteins and aconitase [74].

\section{Antioxidative actions}

The long-chain $n$-3 PUFAs in fish oil, especially EPA and DHA, have well-known antioxidant properties [75]. Recent studies showed that 3 weeks of a diet enriched in $n-3$ PUFAs was sufficient to cause increased antioxidant enzyme expression and decreased mitochondrial ROS generation in mouse heart [76] and human heart [77]. These findings implicate peroxisome-proliferator-activated receptor $\gamma$ activation and augmented mitochondrial fatty acid oxidation as possible therapeutic mechanisms of $n-3$ PUFAs, and suggest that $n$-3 PUFAs exert beneficial effects by enhancing antioxidant capacity. Providing more than $3 \mathrm{~g}$ of marine $n$-3 PUFAs per day to healthy volunteers resulted in decreased production of ROS by blood neutrophils stimulated with different agents [78] and high-dose marine $n$-3 PUFAs decreased hydrogen peroxide production by human monocytes [79]. Rees et al. [80] identified an EPA-dose-dependent decrease in the number of blood neutrophils producing superoxide in elderly subjects, and therefore fish oil supplements decreased ROS production by neutrophils. In addition, DHA and EPA showed antioxidative properties through activation of nuclear-factorlike 2, accompanied by induction of antioxidant enzymes such as heme oxygenase 1 , which may explain the mechanism of the cytoprotective effects of DHA and EPA [81]. There have been a number of clinical trials of fish oil in patients with inflammation. Most of these trials report some clinical improvements-for example, improved patientassessed pain, decreased morning stiffness, fewer painful or tender joints, and decreased use of NSAIDs - and when the trials have been pooled in meta-analyses, statistically significant clinical benefit has emerged.

\section{Cell fluidity and lipid raft changes}

The molecular basis of both the therapeutic benefit of and the pathogenesis caused by NSAIDs has been attributed to the ability of these drugs to inhibit COX, the rate-limiting enzyme in the biosynthesis of a family of prostaglandins, which plays a central role in the mediation of inflammatory, nociceptive, and cytoprotective cellular pathways [82]. One of the alternative mechanisms by which NSAIDs can affect both biological and cytotoxic responses is by interacting with cellular membranes and altering their biophysical properties [83]. NSAIDs are highly amphiphilic and strongly associate with lipid membranes, which influence localization, structure, and function of membrane-associating proteins and actively regulate cell signaling events $[84,85]$. Thus, it is possible that NSAIDs regulate diverse cell functions by altering microenvironments within the membrane through changes in the fluidity, permeability, and biomechanical properties of cell membranes. The interaction of NSAIDs with phospholipid and the ability of the drugs to partition into the membranes can induce marked changes in the permeability, fluidity, and biomechanical properties of these membranes [83-85], after which these NSAID-phospholipid-induced changes will not only increase the permeability of biological membranes to potentially damaging ions, $\mathrm{H}^{+}$and macromolecules, e.g., enterotoxins, but will also induce an instability of the membranes owing to the formation of unstable pores as indicated by a decrease in lysis [86]. Thus, this chemical association between NSAIDs and zwitterionic phospholipids provides us with a molecular understanding of the pH-dependent effects of this potent class of anti-inflammatory drugs to induce injury to the GI 
mucosa, resulting in ulcer formation and bleeding, which may lead to an increased risk of developing potentially lifethreatening hemorrhage. Nunes et al. [87] demonstrated that in general NSAIDs interact with polar head groups of the membrane phospholipids when the drugs are charged and penetrate deeper into the membrane when the drugs are neutral and may result in the development of pores in the bilayer. Further, they demonstrated that these interactions appear to be dependent on the lipid phase [88]. One central molecular target of $n-3$ PUFAs involves alterations to the plasma membrane. DHA has been shown to have significant effects on plasma membrane properties, including altering membrane fluidity, phase behavior, permeability, fusion, flip-flop, and resident protein activity [89, 90]. Furthermore, recent evidence suggests that DHA can perturb specialized regions of the plasma membrane known as lipid rafts [91-93]. Lipid rafts are small, 10-200-nm, heterogeneous microdomains that are enriched in cholesterol, sphingolipids, and saturated acyl chains [94]. Lipid rafts serve as signaling platforms by compartmentalizing plasma membrane proteins and lipids. In response to stimuli, nanometer-scale domains can coalesce and display high molecular order [95]. Signaling pathways emanating from lipid rafts mediate a variety of mitogenic, metastatic, and other tumor-promoting cellular activities, and these pathways are often hyperactivated in cancer [96]. Additionally, chronic inflammation, central to the process of tumorigenesis [97], involves excessive T-cell activation, which is regulated by lipid rafts. The property of $n-3$ PUFAs in the regulation of lipid rafts, and lipid-raft-mediated signaling may protect cells against NSAID-induced changes in membrane composition resulting in altered cell function. $n$ 3 PUFAs enhanced the clustering of lipid rafts to form large raft domains [92] and induced modifications of lipid raft composition [98, 99], which are significant because cholesterol and sphingomyelin are major building blocks of lipid rafts that promote the formation of hydrophobicliquid-ordered molecular packing. In addition, lipid raft organization modified by $n$-3 PUFAs, i.e., altered the physical properties of biological membranes, leads to altered cell signaling and function [100, 101]. These studies suggest a specificity of $n-3$ PUFAs in disruption of lipid rafts by NSAIDs. However, further work is needed to clarify the effects of EPA and DHA on lipid raft perturbation.

\section{Perspective of " $n-3$ PUFA based NSAID" development to cover GI and CV risk of NSAIDs}

When NSAIDs associate with surface phospholipids, the hydrophobic barrier becomes hydrophilic, allowing acid to permeate the mucosal lining, resulting in disruption of mucosal integrity. As the cells lining the stomach acidify, surface and intracellular membranes break down. With recurrent insult, cells continue to die, resulting in gastric erosions and ulcers. If these NSAID-induced mucosal lesions occur adjacent to an underlying blood vessel, bleeding ulcers can result in potentially life-threatening episodes of hemorrhage. One approach to attenuating the damaging action of NSAIDs on the surface of the GI mucosa, which is caused by the drugs interacting with phospholipids present either in the bilayer or in the presumptive monolayer coating the mucus gel lining, is to preassociate the NSAID with either synthetic or natural phosphatidylcholine. Lichtenberger et al. [86, 102] developed a noncovalent complex between natural phosphatidylcholine and an NSAID. The association of the NSAID and phosphatidylcholine appears to render the drug more lipophilic and facilitates its transit across the GI mucosa while minimizing surface injury, with no loss in functional bioavailability and therapeutic efficacy [83]. This approach reduces the affinity of a luminal NSAID to interact with intrinsic phospholipids of the GI mucosa, fortifying the tissue's barrier properties, while promoting the transit of the drug across the mucosa into the blood owing the increased lipophilicity of the conjugate. This appears to be an effective strategy to reduce the GI toxicity of a number of NSAIDs, notably aspirin, ibuprofen, naproxen, and indomethacin, while maintaining their bioavailability and therapeutic efficacy in both rodent models and pilot clinical endoscopic trials $[102,103]$. In this review, we have presented the beneficial effects of $n-3$ PUFAs on NSAIDinduced apoptosis, inflammation, and oxidative stress. Therefore, the development of $n$-3 PUFA based NSAIDs or a coadministration regimen may guarantee clinical safety of NSAID administration, the efficacy of which might soon be potentiated by the introduction of a gastroretentive drug delivery system (Fig. 3).

\section{Conclusion}

To rescue patients from NSAID-induced GI adverse effects, several approaches had been adopted, including coxibs, co-medication of PPI or synthetic prostaglandin analogs, and efficacious phytoceuticals [104]. Recently, newer NSAIDs to alleviate GI and CV pitfalls inherent to current therapy options across the entire NSAID class are mandatory and are under active development. In detail, NO- or $\mathrm{H}_{2} \mathrm{~S}$-releasing NSAIDs, phosphatidylcholine-combined formula, injectable formulation, potent natural phytoceuticals, two orally administered NSAID formulations (e.g., naproxen plus PPI or ibuprofen plus oxycodone), three topical NSAID formulations (e.g., diclofenac patch, gel, and solution), novel intra-articular extended-release 
NSAIDs, co-prescription of gastroprotective agents, and nanoformulations of submicron NSAIDs featuring delivery of decreased doses will be the future of NSAID therapy [105-107]. Our investigation group strongly believes our novel formula, $n-3$ PUFA based NSAIDs, can achieve (1) an NSAID-sparing effect based on the authentic antiinflammatory action of $n-3$ PUFAs, (2) guaranteed safety of GI, CV, and renal systems, (3) correction of all derangements relevant to NSAIDs, (4) significant protection of the whole GI tract, and (5) concerted mode of action against arthritis. However, more purified and concentrated $n-3$ PUFAs should be generated to successfully support the authentic action of NSAIDs. Since the in-hospital case fatality for upper and lower GI tract complication events has remained constant despite the new therapeutic and prevention strategies [6], more effort to develop more efficacious but safer NSAIDs should be pursued in the clinic in the era of the super aging society.

Acknowledgment This research was supported by the National Research Foundation of Korea funded by the Ministry of Science, ICT and Future Planning (2012-0168).

Conflict of interest The authors declare that they have no conflict of interest.

\section{References}

1. Curiel RV, Katz JD. Mitigating the cardiovascular and renal effects of NSAIDs. Pain Med. 2013;14(Suppl 1):S23-8.

2. Vonkeman HE, van de Laar MA. Nonsteroidal anti-inflammatory drugs: adverse effects and their prevention. Semin Arthritis Rheum. 2010;39(4):294-312.

3. Gargallo CJ, Sostres C, Lanas A. Prevention and treatment of NSAID gastropathy. Curr Treat Options Gastroenterol. 2014;12(4):398-413.

4. Scheiman JM, Hindley CE. Strategies to optimize treatment with NSAIDs in patients at risk for gastrointestinal and cardiovascular adverse events. Clin Ther. 2010;32(4):667-77.

5. Sostres C, Gargallo CJ, Lanas A. Interaction between Helicobacter pylori infection, nonsteroidal anti-inflammatory drugs and/or low-dose aspirin use: old question new insights. World J Gastroenterol. 2014;20(28):9439-50.

6. Sostres C, Gargallo CJ, Lanas A. Nonsteroidal anti-inflammatory drugs and upper and lower gastrointestinal mucosal damage. Arthritis Res Ther. 2013;15(Suppl 3):S3.

7. Wallace JL, Syer S, Denou E, de Palma G, Vong L, McKnight $\mathrm{W}$, et al. Proton pump inhibitors exacerbate NSAID-induced small intestinal injury by inducing dysbiosis. Gastroenterology. 2011;141(4):1314-22.e5.

8. Targownik LE, Metge CJ, Leung S, Chateau DG. The relative efficacies of gastroprotective strategies in chronic users of nonsteroidal anti-inflammatory drugs. Gastroenterology. 2008; 134(4):937-44.

9. Coruzzi G, Venturi N, Spaggiari S. Gastrointestinal safety of novel nonsteroidal antiinflammatory drugs: selective COX-2 inhibitors and beyond. Acta Biomed. 2007;78(2):96-110.

10. Singh G. Recent considerations in nonsteroidal anti-inflammatory drug gastropathy. Am J Med. 1998;105(1B):31S-8S.
11. Conaghan PG. A turbulent decade for NSAIDs: update on current concepts of classification, epidemiology, comparative efficacy, and toxicity. Rheumatol Int. 2012;32(6):1491-502.

12. Weir MR. Renal effects of nonselective NSAIDs and coxibs. Cleveland Clin J Med. 2002;69(Suppl 1):SI53-8.

13. Ejaz P, Bhojani K, Joshi VR. NSAIDs and kidney. J Assoc Phys India. 2004;52:632-40.

14. Sandoval-Acuna C, Lopez-Alarcon C, Aliaga ME, Speisky H. Inhibition of mitochondrial complex I by various non-steroidal anti-inflammatory drugs and its protection by quercetin via a coenzyme Q-like action. Chem Biol Interact. 2012;199(1):18-28.

15. Li H, Hortmann M, Daiber A, Oelze M, Ostad MA, Schwarz $\mathrm{PM}$, et al. Cyclooxygenase 2-selective and nonselective nonsteroidal anti-inflammatory drugs induce oxidative stress by upregulating vascular NADPH oxidases. J Pharmacol Exp Ther. 2008;326(3):745-53.

16. Sharma JN, Jawad NM. Adverse effects of COX-2 inhibitors. Sci World J. 2005;5:629-45.

17. Fiorucci S, Santucci L, Distrutti E. NSAIDs, coxibs, CINOD and $\mathrm{H}_{2} \mathrm{~S}$-releasing NSAIDs: what lies beyond the horizon. Dig Liver Dis. 2007;39(12):1043-51.

18. Fiorucci S, Santucci L. Hydrogen sulfide-based therapies: focus on $\mathrm{H} 2 \mathrm{~S}$ releasing NSAIDs. Inflamm Allergy Drug Targets. 2011;10(2):133-40.

19. Bertolini A, Ottani A, Sandrini M. Dual acting anti-inflammatory drugs: a reappraisal. Pharmacol Res. 2001;44(6):437-50.

20. Leone S, Ottani A, Bertolini A. Dual acting anti-inflammatory drugs. Curr Top Med Chem. 2007;7(3):265-75.

21. Celotti F, Laufer S. Anti-inflammatory drugs: new multitarget compounds to face an old problem. The dual inhibition concept. Pharmacol Res. 2001;43(5):429-36.

22. Kangwan N, Park JM, Hahm KB. Development of GI-safe NSAID; progression from the bark of willow tree to modern pharmacology. Curr Opin Pharmacol. 2014;19C:17-23.

23. Kang JX, Wang J, Wu L, Kang ZB. Transgenic mice: fat-1 mice convert n-6 to n-3 fatty acids. Nature. 2004;427(6974):504.

24. Kang JX. Fat-1 transgenic mice: a new model for omega-3 research. Prostaglandins Leukot Essent Fatty Acids. 2007; 77(5-6):263-7.

25. Zheng H, Tang H, Liu M, He M, Lai P, Dong H, et al. Inhibition of endometrial cancer by $\mathrm{n}-3$ polyunsaturated fatty acids in preclinical models. Cancer Prev Res (Phila). 2014;7(8):824-34.

26. Weylandt KH, Krause LF, Gomolka B, Chiu CY, Bilal S, Nadolny A, et al. Suppressed liver tumorigenesis in fat-1 mice with elevated omega-3 fatty acids is associated with increased omega- 3 derived lipid mediators and reduced TNF- $\alpha$. Carcinogenesis. 2011;32(6):897-903.

27. Xia S, Lu Y, Wang J, He C, Hong S, Serhan CN, et al. Melanoma growth is reduced in fat- 1 transgenic mice: impact of omega-6/omega-3 essential fatty acids. Proc Natl Acad Sci U S A. 2006;103(33):12499-504.

28. Li J, Li FR, Wei D, Jia W, Kang JX, Stefanovic-Racic M, et al. Endogenous omega-3 polyunsaturated fatty acid production confers resistance to obesity, dyslipidemia, and diabetes in mice. Mol Endocrinol. 2014;28(8):1316-28.

29. Bellenger J, Bellenger S, Bataille A, Massey KA, Nicolaou A, Rialland M, et al. High pancreatic n-3 fatty acids prevent STZinduced diabetes in fat-1 mice: inflammatory pathway inhibition. Diabetes. 2011;60(4):1090-9.

30. Cai A, Hutchison E, Hudson J, Kawashima Y, Komori N, Singh A, et al. Metabolic enrichment of omega-3 polyunsaturated fatty acids does not reduce the onset of idiopathic knee osteoarthritis in mice. Osteoarthritis Cartilage. 2014;22(9):1301-9.

31. Luo C, Ren H, Wan JB, Yao X, Zhang X, He C, et al. Enriched endogenous omega-3 fatty acids in mice protect against global ischemia injury. J Lipid Res. 2014;55(7):1288-97. 
32. Hu X, Zhang F, Leak RK, Zhang W, Iwai M, Stetler RA, et al. Transgenic overproduction of omega- 3 polyunsaturated fatty acids provides neuroprotection and enhances endogenous neurogenesis after stroke. Curr Mol Med. 2013;13(9):1465-73.

33. Lopez-Vicario C, Gonzalez-Periz A, Rius B, Moran-Salvador E, Garcia-Alonso V, Lozano JJ, et al. Molecular interplay between $\Delta 5 / \Delta 6$ desaturases and long-chain fatty acids in the pathogenesis of non-alcoholic steatohepatitis. Gut. 2014;63(2):344-55.

34. Kim EH, Bae JS, Hahm KB, Cha JY. Endogenously synthesized $\mathrm{n}-3$ polyunsaturated fatty acids in fat- 1 mice ameliorate high-fat diet-induced non-alcoholic fatty liver disease. Biochem Pharmacol. 2012;84(10):1359-65.

35. Lim K, Han C, Dai Y, Shen M, Wu T. Omega-3 polyunsaturated fatty acids inhibit hepatocellular carcinoma cell growth through blocking $\beta$-catenin and cyclooxygenase- 2 . Mol Cancer Ther. 2009;8(11):3046-55.

36. Tsutsumi S, Gotoh T, Tomisato W, Mima S, Hoshino T, Hwang $\mathrm{HJ}$, et al. Endoplasmic reticulum stress response is involved in nonsteroidal anti-inflammatory drug-induced apoptosis. Cell Death Differ. 2004;11(9):1009-16.

37. Levine B, Kroemer G. Autophagy in the pathogenesis of disease. Cell. 2008;132(1):27-42.

38. Chen ST, Thomas S, Gaffney KJ, Louie SG, Petasis NA, Schonthal AH. Cytotoxic effects of celecoxib on Raji lymphoma cells correlate with aggravated endoplasmic reticulum stress but not with inhibition of cyclooxygenase-2. Leuk Res. 2010;34(2): 250-3.

39. Chiou SK, Tanigawa T, Akahoshi T, Abdelkarim B, Jones MK, Tarnawski AS. Survivin: a novel target for indomethacininduced gastric injury. Gastroenterology. 2005;128(1):63-73.

40. Chiou SK, Mandayam S. NSAIDs enhance proteasomic degradation of survivin, a mechanism of gastric epithelial cell injury and apoptosis. Biochem Pharmacol. 2007;74(10):1485-95.

41. Farrugia G, Balzan R. The proapoptotic effect of traditional and novel nonsteroidal anti-inflammatory drugs in mammalian and yeast cells. Oxid Med Cell Longev. 2013;2013:504230.

42. Schwab JM, Serhan CN. Lipoxins and new lipid mediators in the resolution of inflammation. Curr Opin Pharmacol. 2006;6(4): 414-20.

43. Arita M, Clish CB, Serhan CN. The contributions of aspirin and microbial oxygenase to the biosynthesis of anti-inflammatory resolvins: novel oxygenase products from omega-3 polyunsaturated fatty acids. Biochem Biophys Res Commun. 2005;338(1):149-57.

44. Serhan CN, Hong S, Gronert K, Colgan SP, Devchand PR, Mirick G, et al. Resolvins: a family of bioactive products of omega-3 fatty acid transformation circuits initiated by aspirin treatment that counter proinflammation signals. J Exp Med. 2002;196(8):1025-37.

45. Spite M, Serhan CN. Novel lipid mediators promote resolution of acute inflammation: impact of aspirin and statins. Circ Res. 2010;107(10):1170-84.

46. Sun YP, Oh SF, Uddin J, Yang R, Gotlinger K, Campbell E, et al. Resolvin D1 and its aspirin-triggered 17R epimer. Stereochemical assignments, anti-inflammatory properties, and enzymatic inactivation. J Biol Chem. 2007;282(13):9323-34.

47. Jung TW, Hwang HJ, Hong HC, Choi HY, Yoo HJ, Baik SH, et al. Resolvin D1 reduces ER stress-induced apoptosis and triglyceride accumulation through JNK pathway in HepG2 cells. Mol Cell Endocrinol. 2014;391(1-2):30-40.

48. Ohta M, Kawano H, Notsu T, Naba H, Imada K. Eicosapentaenoic acid attenuates statin-induced ER stress and toxicity in myoblast. Biochem Biophys Res Commun. 2012;424(2):301-7.

49. Begum G, Yan HQ, Li L, Singh A, Dixon CE, Sun D. Docosahexaenoic acid reduces ER stress and abnormal protein accumulation and improves neuronal function following traumatic brain injury. J Neurosci. 2014;34(10):3743-55.

50. Lanza FL. A review of gastric ulcer and gastroduodenal injury in normal volunteers receiving aspirin and other non-steroidal antiinflammatory drugs. Scand J Gastroenterol Suppl. 1989;163: 24-31.

51. Wallace JL. Nonsteroidal anti-inflammatory drugs and gastroenteropathy: the second hundred years. Gastroenterology. 1997;112(3):1000-16.

52. Wallace JL, Vong L. NSAID-induced gastrointestinal damage and the design of GI-sparing NSAIDs. Curr Opin Investig Drugs. 2008;9(11):1151-6.

53. Faust T, Redfern JS, Lee E, Feldman M. Effects of fish oil on gastric mucosal 6-keto-PGF1 alpha synthesis and ethanolinduced injury. Am J Physiol. 1989;257(1 Pt 1):G9-13.

54. Leung FW. Prostaglandins mediate fish oil protection against ethanol-induced gastric mucosal injury in rats. Dig Dis Sci. 1994;39(4):893.

55. Faust TW, Redfern JS, Podolsky I, Lee E, Grundy SM, Feldman M. Effects of aspirin on gastric mucosal prostaglandin E2 and F2 alpha content and on gastric mucosal injury in humans receiving fish oil or olive oil. Gastroenterology. 1990;98(3): 586-91.

56. Bhattacharya A, Ghosal S, Bhattacharya SK. Effect of fish oil on offensive and defensive factors in gastric ulceration in rats. Prostaglandins Leukot Essent Fatty Acids. 2006;74(2):109-16.

57. Guzel C, Ulak G, Sermet A, Cicek R, Ulak M. Effect of fish oil on indometacin-induced gastric lesions in rats. Arzneimittelforschung. 1995;45(11):1172-3.

58. Manjari V, Das UN. Effect of polyunsaturated fatty acids on dexamethasone-induced gastric mucosal damage. Prostaglandins Leukot Essent Fatty Acids. 2000;62(2):85-96.

59. Ulak G, Cicek R, Sermet A, Guzel C, Ulak M, Denli O. Protective effect of fish oil against stress-induced gastric injury in rats. Arzneimittelforschung. 1995;45(11):1174-5.

60. Mayurasakorn K, Williams JJ, Ten VS, Deckelbaum RJ. Docosahexaenoic acid: brain accretion and roles in neuroprotection after brain hypoxia and ischemia. Curr Opin Clin Nutr Metab Care. 2011;14(2):158-67.

61. Saravanan P, Davidson NC, Schmidt EB, Calder PC. Cardiovascular effects of marine omega-3 fatty acids. Lancet. 2010;376(9740):540-50.

62. Cho JY, Chi SG, Chun HS. Oral administration of docosahexaenoic acid attenuates colitis induced by dextran sulfate sodium in mice. Mol Nutr Food Res. 2011;55(2):239-46.

63. Asako H, Kubes P, Wallace J, Gaginella T, Wolf RE, Granger DN. Indomethacin-induced leukocyte adhesion in mesenteric venules: role of lipoxygenase products. Am $\mathrm{J}$ Physiol. 1992;262(5 Pt 1):G903-8.

64. Santucci L, Fiorucci S, Giansanti M, Brunori PM, Di Matteo FM, Morelli A. Pentoxifylline prevents indomethacin induced acute gastric mucosal damage in rats: role of tumour necrosis factor alpha. Gut. 1994;35(7):909-15.

65. Pineda-Pena EA, Jimenez-Andrade JM, Castaneda-Hernandez G, Chavez-Pina AE. Docosahexaenoic acid, an omega-3 polyunsaturated acid protects against indomethacin-induced gastric injury. Eur J Pharmacol. 2012;697(1-3):139-43.

66. Gil A. Polyunsaturated fatty acids and inflammatory diseases. Biomed Pharmacother. 2002;56(8):388-96.

67. Simopoulos AP. Omega-3 fatty acids in inflammation and autoimmune diseases. J Am Coll Nutr. 2002;21(6):495-505.

68. Fernandes E, Toste SA, Lima JL, Reis S. The metabolism of sulindac enhances its scavenging activity against reactive oxygen and nitrogen species. Free Radic Biol Med. 2003;35(9): 1008-17. 
69. Costa D, Gomes A, Reis S, Lima JL, Fernandes E. Hydrogen peroxide scavenging activity by non-steroidal anti-inflammatory drugs. Life Sci. 2005;76(24):2841-8.

70. Yiannakopoulou E, Tiligada E. Protective effect of salicylates against hydrogen peroxide stress in yeast. J Appl Microbiol. 2009;106(3):903-8.

71. Herrero E, Ros J, Belli G, Cabiscol E. Redox control and oxidative stress in yeast cells. Biochim Biophys Acta. 2008; 1780(11):1217-35.

72. Oh KW, Qian T, Brenner DA, Lemasters JJ. Salicylate enhances necrosis and apoptosis mediated by the mitochondrial permeability transition. Toxicol Sci. 2003;73(1):44-52.

73. Battaglia V, Salvi M, Toninello A. Oxidative stress is responsible for mitochondrial permeability transition induction by salicylate in liver mitochondria. J Biol Chem. 2005; 280(40):33864-72.

74. Raza H, John A, Benedict S. Acetylsalicylic acid-induced oxidative stress, cell cycle arrest, apoptosis and mitochondrial dysfunction in human hepatoma HepG2 cells. Eur J Pharmacol. 2011;668(1-2):15-24.

75. Giordano E, Visioli F. Long-chain omega 3 fatty acids: molecular bases of potential antioxidant actions. Prostaglandins Leukot Essent Fatty Acids. 2014;90(1):1-4.

76. Anderson EJ, Thayne K, Harris M, Carraway K, Shaikh SR. Aldehyde stress and up-regulation of Nrf2-mediated antioxidant systems accompany functional adaptations in cardiac mitochondria from mice fed n-3 polyunsaturated fatty acids. Biochem J. 2012;441(1):359-66.

77. Anderson EJ, Thayne KA, Harris M, Shaikh SR, Darden TM, Lark DS, et al. Do fish oil omega-3 fatty acids enhance antioxidant capacity and mitochondrial fatty acid oxidation in human atrial myocardium via PPAR $\gamma$ activation? Antioxid Redox Signal. 2014;21(8):1156-63.

78. Varming K, Schmidt EB, Svaneborg N, Moller JM, Lervang $\mathrm{HH}$, Grunnet N, et al. The effect of n-3 fatty acids on neutrophil chemiluminescence. Scand J Clin Lab Invest. 1995;55(1):47-52.

79. Fisher M, Levine PH, Weiner BH, Johnson MH, Doyle EM, Ellis PA, et al. Dietary n-3 fatty acid supplementation reduces superoxide production and chemiluminescence in a monocyteenriched preparation of leukocytes. Am J Clin Nutr. 1990;51(5):804-8.

80. Rees D, Miles EA, Banerjee T, Wells SJ, Roynette CE, Wahle $\mathrm{KW}$, et al. Dose-related effects of eicosapentaenoic acid on innate immune function in healthy humans: a comparison of young and older men. Am J Clin Nutr. 2006;83(2):331-42.

81. Ishikado A, Morino K, Nishio Y, Nakagawa F, Mukose A, Sono Y, et al. 4-Hydroxy hexenal derived from docosahexaenoic acid protects endothelial cells via Nrf2 activation. PLoS One. 2013;8(7):e69415.

82. Flower RJ, Vane JR. Inhibition of prostaglandin synthetase in brain explains the anti-pyretic activity of paracetamol (4-acetamidophenol). Nature. 1972;240(5381):410-1.

83. Lichtenberger LM, Wang ZM, Romero JJ, Ulloa C, Perez JC, Giraud MN, et al. Non-steroidal anti-inflammatory drugs (NSAIDs) associate with zwitterionic phospholipids: insight into the mechanism and reversal of NSAID-induced gastrointestinal injury. Nat Med. 1995;1(2):154-8.

84. Manrique-Moreno M, Garidel P, Suwalsky M, Howe J, Brandenburg K. The membrane-activity of ibuprofen, diclofenac, and naproxen: a physico-chemical study with lecithin phospholipids. Biochim Biophys Acta. 2009;1788(6):1296-303.

85. Giraud MN, Motta C, Romero JJ, Bommelaer G, Lichtenberger LM. Interaction of indomethacin and naproxen with gastric surface-active phospholipids: a possible mechanism for the gastric toxicity of nonsteroidal anti-inflammatory drugs (NSAIDs). Biochem Pharmacol. 1999;57(3):247-54.
86. Lichtenberger LM, Zhou Y, Dial EJ, Raphael RM. NSAID injury to the gastrointestinal tract: evidence that NSAIDs interact with phospholipids to weaken the hydrophobic surface barrier and induce the formation of unstable pores in membranes. J Pharm Pharmacol. 2006;58(11):1421-8.

87. Nunes C, Brezesinski G, Lima JL, Reis S, Lucio M. Synchrotron SAXS and WAXS study of the interactions of NSAIDs with lipid membranes. J Phys Chem B. 2011;115(24):8024-32.

88. Nunes C, Brezesinski G, Pereira-Leite C, Lima JL, Reis S, Lucio M. NSAIDs interactions with membranes: a biophysical approach. Langmuir ACS J Surfaces Colloids. 2011;27(17): $10847-58$

89. Stillwell W, Wassall SR. Docosahexaenoic acid: membrane properties of a unique fatty acid. Chem Phys Lipids. 2003;126(1):1-27.

90. Wassall SR, Brzustowicz MR, Shaikh SR, Cherezov V, Caffrey M, Stillwell W. Order from disorder, corralling cholesterol with chaotic lipids. The role of polyunsaturated lipids in membrane raft formation. Chem Phys Lipids. 2004;132(1):79-88.

91. Chapkin RS, McMurray DN, Davidson LA, Patil BS, Fan YY, Lupton JR. Bioactive dietary long-chain fatty acids: emerging mechanisms of action. Br J Nutr. 2008;100(6):1152-7.

92. Kim W, Fan YY, Barhoumi R, Smith R, McMurray DN, Chapkin RS. n-3 polyunsaturated fatty acids suppress the localization and activation of signaling proteins at the immunological synapse in murine CD4 $+\mathrm{T}$ cells by affecting lipid raft formation. J Immunol. 2008;181(9):6236-43.

93. Shaikh SR, Rockett BD, Salameh M, Carraway K. Docosahexaenoic acid modifies the clustering and size of lipid rafts and the lateral organization and surface expression of MHC class I of EL4 cells. J Nutr. 2009;139(9):1632-9.

94. Pike LJ. Rafts defined: a report on the keystone symposium on lipid rafts and cell function. J Lipid Res. 2006;47(7):1597-8.

95. Lingwood D, Simons K. Lipid rafts as a membrane-organizing principle. Science. 2010;327(5961):46-50.

96. Patra SK. Dissecting lipid raft facilitated cell signaling pathways in cancer. Biochim Biophys Acta. 2008;1785(2):182-206.

97. Ullman TA, Itzkowitz SH. Intestinal inflammation and cancer. Gastroenterology. 2011;140(6):1807-16.

98. Ma DW, Seo J, Davidson LA, Callaway ES, Fan YY, Lupton JR, et al. n-3 PUFA alter caveolae lipid composition and resident protein localization in mouse colon. FASEB J. 2004;18(9):1040-2.

99. Turk HF, Chapkin RS. Membrane lipid raft organization is uniquely modified by n-3 polyunsaturated fatty acids. Prostaglandins Leukot Essent Fatty Acids. 2013;88(1):43-7.

100. Fan YY, Ly LH, Barhoumi R, McMurray DN, Chapkin RS. Dietary docosahexaenoic acid suppresses $\mathrm{T}$ cell protein kinase $\mathrm{C} \theta$ lipid raft recruitment and IL-2 production. J Immunol. 2004;173(10):6151-60.

101. Ben-Neriah Y, Karin M. Inflammation meets cancer, with NF$\kappa \mathrm{B}$ as the matchmaker. Nat Immunol. 2011;12(8):715-23.

102. Cryer B, Bhatt DL, Lanza FL, Dong JF, Lichtenberger LM, Marathi UK. Low-dose aspirin-induced ulceration is attenuated by aspirin-phosphatidylcholine: a randomized clinical trial. Am J Gastroenterol. 2011;106(2):272-7.

103. Anand BS, Romero JJ, Sanduja SK, Lichtenberger LM. Phospholipid association reduces the gastric mucosal toxicity of aspirin in human subjects. Am J Gastroenterol. 1999;94(7): $1818-22$.

104. Sinha M, Gautam L, Shukla PK, Kaur P, Sharma S, Singh TP. Current perspectives in NSAID-induced gastropathy. Mediators Inflamm. 2013;2013:258209.

105. Argoff CE. Recent developments in the treatment of osteoarthritis with NSAIDs. Curr Med Res Opin. 2011;27(7): 1315-27. 
106. Atkinson TJ, Fudin J, Jahn HL, Kubotera N, Rennick AL, Rhorer M. What's new in NSAID pharmacotherapy: oral agents to injectables. Pain Med. 2013;14(Suppl 1):S11-7.

107. Medlock S, Eslami S, Askari M, Taherzadeh Z, Opondo D, de Rooij SE, et al. Co-prescription of gastroprotective agents and their efficacy in elderly patients taking nonsteroidal antiinflammatory drugs: a systematic review of observational studies. Clin Gastroenterol Hepatol. 2013;11(10):1259-69.e10. 